COMMUNITY CHOICE

The most viewed papers in science

\title{
CHEMISTRY
}

\section{Iron nanoparticles into blood}

proteins that are not mutated or overexpressed may also be crucial to a cancer cell's ability to survive.

Christopher Lord and his team at the Institute of Cancer Research in London used RNA interference screening which silences individual genes by interfering with protein breast-cancer cell lines. These lines represented all the major subtypes of the disease, offering a chance to find tumourspecific vulnerabilities and potential drug targets.

The authors uncovered a series of novel Achilles heels for particular tumour cell lines, including the reliance of cells deficient in the protein PTEN on TTK kinase, and the sensitivity of oestrogenreceptor-positive breastcancer cells to the loss of the kinase ADCK2.

Cancer Discov. http:/dx.doi.org/ (2011)

\section{MATERIALS}

\section{Butterfly wings turned to metal}

The tiny scales that lend colour and texture to a butterfly's wings can be used as organic templates to make metallic nanostructures (pictured).

Jiajun Gu, Di Zhang and their colleagues at Shanghai Jiao Tong University in China first added gold nanoparticles to the surface of the wing scales, which are made from the tough polysaccharide chitin. They then deposited a metallic coating on the structures before The large variety of butterflywing morphologies means that every butterfly bears scales of different shapes and size.

The authors' fabrication method is adaptable to at least synthesis - on a panel of 20 10.1158/2159-8290.CD-11-0107 removing the organic material. seven common metals. With almost 175,000 species of butterfly and moth to choose from, materials scientists could generate a wealth of intact, three-dimensional shapes with submicrometre resolution. The nanostructures have many potential applications - for example, in the design of photonic crystals. Angew. Chem. Int. Edn http://dx.doi.org/10.1002/ anie.201103505 (2011)

\section{ASTROPHYSICS}

\section{Welcome to the neighbourhood}

The Sun's neighbourhood is a little more crowded than we realized. Astronomers have discovered two ultracool brown dwarfs around 5 parsecs away - just four times farther than the distance to our next-nearest star, Proxima Centauri.

Brown dwarfs are objects bigger than Jupiter, but not big enough for their gravity to ignite fusion, as in a star. Ralf-Dieter Scholz and his colleagues at the Leibniz Institute for Astrophysics in Potsdam, Germany, found the brown dwarfs using NASA's Wide-field Infrared Survey Explorer. They looked for objects that had moved a lot in the sky since two previous ground-based surveys, indicating that these objects are close to us.

Astron. Astrophys. 532, L5 (2011) IMMUNOLOGY

\section{An antibody for all flu strains}

A person who contracted the strain of influenza responsible for the 2009-10 pandemic produced an antibody with the remarkable ability to block all strains of influenza A.

\section{HIGHLY READ \\ on pubs.acs.org in July}

Tiny particles of iron oxide can have their surface chemistry manipulated to make them soluble in water and so more useful in medical applications. Iron oxide nanoparticles have potential for drug delivery and as contrast agents for magnetic resonance imaging, but they are generally made in organic solvents, and keeping them stable and soluble in the body's aqueous environment has proved difficult.

Yuping Bao and her colleagues at the University of Alabama in Tuscaloosa found a way to swap the chemical groups originally coating the nanoparticles for groups that make the particles soluble in biological solvents. The process worked best when the original groups were trioctylphosphine oxide and their replacements were poly(acrylic acid), polyethylenimine or glutathione - provided at a ratio of five to each nanoparticle. Langmuir 27, 8990-8997 (2011)
Antonio Lanzavecchia at the Institute for Research in Biomedicine in Bellinzona, Switzerland, John Skehel of the MRC National Institute for Medical Research in London and their team scoured 104,000 white blood cells from eight people. They identified four $B$ cells from one donor that churn out an antibody, dubbed FI6, which recognizes the haemagglutinin coat protein made by all 16 subtypes of influenza A viruses. Mice and ferrets given FI6 or a modified version of it survived infection with different flu strains that killed untreated animals.

The crystal structure of the antibody when bound to different haemagglutinin proteins offers a target for the design of a universal flu vaccine able to spark a similar immune response, the authors say.

Science http://dx.doi.org/ 10.1126/science.1205669 (2011) See go.nature.com/naecyt for more on this research.

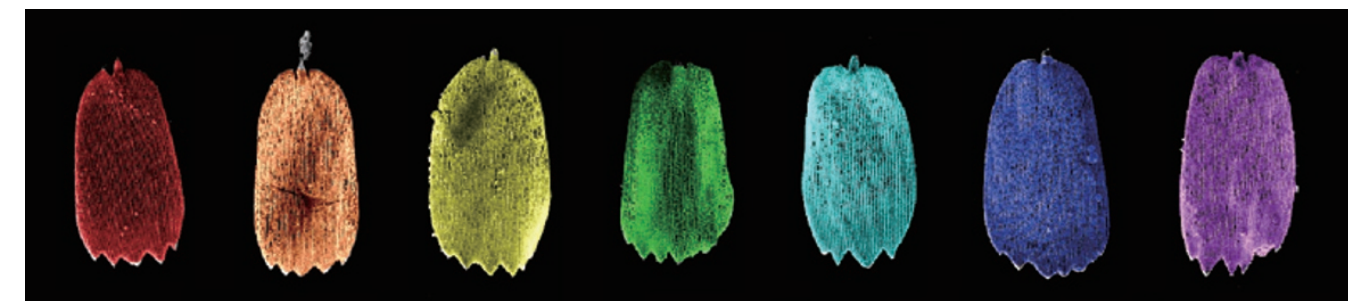

\section{ECOLOGY \\ Pollination drives floral clock}

The closure of flower heads is driven not only by falling light or temperature, as previously thought, but also by pollinators In a set of field experiments, Jochen Fründ at Georg August University in Göttingen, Germany, and his team show that flowers in the Asteraceae family, including smooth hawksbeard (Crepis capillaris), close within three hours of pollination. By contrast, the heads of unpollinated flowers stayed open for hours longer until late afternoon.

Flower-head closure earlier in the day affects interactions between plants and the pollinators of the surrounding area, and can change the times at which some insects are active. The authors suggest that their results be considered in plant and pollinator field surveys to ensure accuracy. Ecol. Lett. http://dx.doi.org/ 10.1111/j.1461-

0248.2011.01654.x (2011)

\section{$\rightarrow$ NATURE.COM}

For the latest research published by Nature visit:

www.nature.com/latestresearch 